\title{
Boundary Element Methods in Elastography - A First Explorative Study
}

\author{
Hans-Uwe Berger $^{a}$, Chris E. Hann ${ }^{a}$, J. Geoffrey Chase ${ }^{a}$, \\ Richard L. Broughton ${ }^{b}$, Elijah Van Houten ${ }^{a}$ \\ ${ }^{a}$ Department of Mechanical Engineering, University of Canterbury, Private Bag 4800, \\ Christchurch 8020, New Zealand; \\ ${ }^{b}$ Department of Mathematics and Statistics, University of Canterbury, Private Bag 4800, \\ Christchurch 8020, New Zealand
}

\begin{abstract}
Next to Magnet Resonance Elastography and Ultrasound Elastography, Digital Image Elasto-Tomography (DIET) is a new imaging-technique, using only motion data available on the boundary, to reconstruct mechanical material parameters, i.e. the interior stiffness of a domain, in order to diagnose tissue related disease such as breast cancer. Where classically Finite Element Methods have been employed to solve this inverse problem, this paper explores a new approach to the reconstruction of mechanical material properties of tissue and tissue defects by the use of Boundary Element Methods (BEM). Using the Boundary Integral Equations for Linear Elasticity in two dimensions within a Conjugate Gradients based inverse solver, material properties of healthy and malicious tissue could be determined from displacement data on the boundary. First simulation results are presented.
\end{abstract}

Keywords: Boundary Element Methods, Inverse Problem, Breast Cancer, Elastography, Mechanical Property Reconstruction, Computational Methods

\section{INTRODUCTION}

Recent statistics confirm that Breast Cancer remains a most serious disease that afflicts roughly $10 \%$ of the female population worldwide, with fatalities in between $10 \%$ to $40 \%$ of all cases. ${ }^{1,2}$ However, early detection, as currently performed by standard screening methods, Palpation and Mammography, can be a significant factor, increasing the five-year survival rate of Breast Cancer patients to $95 \% .^{1,3,4}$

In this field of breast cancer screening, elastographic methods have recently demonstrated success in diagnosing breast cancer lesions. ${ }^{5,6}$ These techniques image the mechanical stiffness distribution of a soft tissue using motion data. The stiffness herein is a parameter that is directly related to disease and shows a difference of up to one order in magnitude between healthy and cancerous tissue. ${ }^{7,8}$ The traditional fields of Elastography are named by the imaging method that supplies the source of motion, Ultrasound (USE) and Magnet Resonance Elastography (MRE). ${ }^{9-11}$

A common drawback to many methods in Elastography is the large computational size of the problem. It has classically been tackled employing finite element methods that determine the forward solution to a problem with a nodally distributed shear modulus. For MR Elastography special techniques, such as the division of the domain into subzones ${ }^{12,13}$ have been developed to deal with the data rich problem.

Digital Image Elasto-Tomography (DIET), as proposed by Chase and Van Houten, ${ }^{14}$ is a novel imaging technique for breast cancer screening. It uses motion data supplied by an array of digital cameras and thus, is only available on the surface of the breast, for the reconstruction of mechanical material parameters throughout the tissue. Peters et al. have demonstrated the capability of the new system by the successful reconstruction of the interior mechanical stiffness distribution using both, simulated data as well as data from measurements

Further author information: (Send correspondence to H.B.)

H.B.: E-mail: hub10@student.canterbury.ac.nz, Telephone: +64 (0)3 3642987 ext. 7092 
on gel phantoms. ${ }^{15-17}$ Even though the DIET system achieves a reduction of the resulting size of the measured data, the resolution of the finite element mesh must remain very fine in order to image lesions of a small size. This still results in an inverse problem of large dimension.

As data from the DIET-method is available only on the surface of the domain, the Boundary Element Method (BEM) appears to be naturally suited to a role in the DIET-system inverse problem. The BEM allows the solution of the underlying PDE for homogeneous regions that are discretized only on their boundary, thus reducing the size of the forward problem. Furthermore, as inhomogeneities need to be handled by regionally specified material properties, a clear differentiation between healthy and cancerous tissue is possible by region and the number of parameters to be reconstructed is reduced immensely.

This paper explores the use of the BEM for two-dimensional linear Elasticity in the static and harmonic case for the solution of different elastographic inverse problems in the DIET system. Embedded in a CG-based nonlinear reconstruction scheme, the BEM in 2D static Elasticity for a single region has been used to determine Poisson's Ratio, $\nu$, where it's harmonic counterpart has been used for the determination of the shear modulus, $\mu$, or Young's modulus, E, respectively (section 3.1). A multiple region based BEM in 2D static Elasticity has also been setup as a body that contains an arbitrarily shaped inclusion of different size, to determine the Poisson Ratios as well as the Elasticity-contrast between the two regions (section 3.2). The measured data was in all cases supplied by forward solves of the BEM, and used for the reconstruction in both noise-free conditions and with uniformly distributed noise of up to $10 \%$ in all of the cases named above.

\section{METHODS}

This section shortly reviews the methods, that have been used for the implementation of the BEM as well as some detail on the reconstruction algorithm with a nonlinear conjugate gradient method.

\subsection{Theory on BEM in Elasticity}

The governing partial differential equation in dynamic linear elasticity is given by Navier's equation in tensor notation in terms of displacements,

$$
(\lambda+\mu) u_{j, j i}+\mu u_{i, j j}+b_{i}=\rho \ddot{u}_{i},
$$

where $\lambda$ and $\mu$ denote Lame's constants, $\mathrm{u}$ the displacements and $b$ the body forces, which are neglected in the following. When the acceleration terms $\ddot{u}$ are set to zero, Equation (1) reduces to the static case of linear Elasticity that could also be written in terms of the body's stress, $\sigma$,

$$
\mu \sigma_{i j, j}=0 .
$$

The boundary element formulation for a homogeneous domain can be either obtained using weighted residuals and application of Green's second theorem or directly from Betti's reciprocal theorem. When displacements are assumed to harmonic, the resulting integral equation is the same in the harmonic and in the static case and can be written for every collocation point $\mathbf{y}$ :

$$
c_{i j}(\mathbf{y}) u_{i}(\mathbf{y})+\int_{\Gamma} T_{i j}(\mathbf{x}, \mathbf{y}) u_{i}(\mathbf{x}) d \Gamma(\mathbf{x})=\int_{\Gamma} U_{i j}(\mathbf{x}, \mathbf{y}) t_{i}(\mathbf{x}) d \Gamma(\mathbf{x}),
$$

where $\Gamma$ denotes the boundary of the given geometry in cartesian coordinates $\mathbf{x} . T_{i j}$ and $U_{i j}$ are the fundamental solutions (Green's functions) to the PDE and are given in the Appendix A for both, the static and the harmonic case. They are used as weighting functions for the integrals over the displacement and traction components $u_{i}$ and $t_{i}$.

For the numerical implementation of equation 3, the boundary is discretized by isoparametric boundary elements, and can be written using a localized coordinate system $\xi$ as

$$
c_{i j}(\mathbf{y}) u_{i}(\mathbf{y})+\sum_{m=1}^{M} \int_{\Gamma_{m}} T_{i j}(\mathbf{x}(\xi), \mathbf{y}) \phi_{k}(\xi) u_{i k} d \Gamma_{m}(\mathbf{x}(\xi))=\sum_{m=1}^{M} \int_{\Gamma_{m}} U_{i j}(\mathbf{x}(\xi), \mathbf{y}) \phi_{k}(\xi) t_{i k} d \Gamma_{m}(\mathbf{x}(\xi)),
$$


where $\phi_{k}(\xi)$ are the nodal shape functions of a boundary element $m=1, \ldots, M$ and $u_{i k}, t_{i k}$ are the nodal values of displacement and traction respectively. Expressing $d \Gamma_{m}$ in terms of the intrinsic coordinate and the element specific Jacobian of the transformation, $d \Gamma_{m}=\left|J_{m}\right| d \xi$, the integrals in Eqn. (4) can be computed by Gaussian Quadrature. Special care has to be taken in the evaluation of the Cauchy principle values in the integral over the traction kernel.

The value of the free term coefficient $c_{i j}$ depends on the geometry at the boundary and is closely related to the problem of corner nodes. In this paper corner nodes were either dealt with by rounding the corner off by some small radius, as for example in the simulations for harmonic Elasticity, or by the use of discontinuous elements. Thus, in all simulations shown, nodes are positioned on a smooth boundary and the free term coefficient is given $c_{i j}=0.5 \delta_{i j}$, with $\delta_{i j}$ being the Kronecker delta.

When the geometry under investigation consists of several materials with different properties, the integral Eqn. (4) needs to be evaluated in the sense of multiple regions. These can then be combined, when the condition of equilibrium and compatibility are satisfied on the interface between the regions, i.e. $t_{I}=-t_{I I}$ and $u_{I}=u_{I I}$.

A full description of the foregoing derivations can be found in the textbooks of P.K. Banerjee ${ }^{18}$ or C.A. Brebbia, ${ }^{19}$ details on it's implementation are found in the textbook of G. Beer. ${ }^{20}$ A description of the BEM for steady state Elastodynamics is found in the textbook by G. Manolis and D. Beskos ${ }^{21}$ and the works by J. Dominguez et al. ${ }^{22}$ and S. Ahmad et al. ${ }^{23}$

\subsection{Reconstruction Algorithm}

Although the the conjugate gradient method (CG) is a commonly used method in many computational problems and would not require further explanations, this section presents a few details relevant to the method of reconstruction of mechanical parameters in the DIET-system.

As a typical gradient descent method, the CG method performs an optimization or reconstruction of an arbitrary parameter $\theta$ based on the minimization of an error function $F$,

$$
\theta=\min _{\theta}\{F(\theta)\},
$$

which in this paper is chosen as the square of a norm over the subtraction between the measured displacements $\tilde{u}$ and simulated displacements $u=f(\theta)$ from the forward solution. The error term can be written as

$$
F=\|\tilde{u}-f(\theta)\|^{2} .
$$

It is important to note here, that $\tilde{u}$ represents only nodal measurements on the free boundary of the outer geometry in question. Although the multiple BEM simulation of an inclusion problem also results in displacement information at the boundary of the interior inclusion, this information cannot be used in the error term, since motion data can only be obtained at the outer surface.

The residual as required in the CG-method is the derivative of Eqn. (6) with respect to $\theta$ and given as

$$
\frac{\partial F}{\partial \theta}=-2\left(\frac{\partial f}{\partial \theta}\right)^{T}\|\tilde{u}-f(\theta)\|,
$$

where the Jacobian $J=\partial f / \partial \theta$ in this application was obtained by a finite difference expression. A NewtonRhapson line search was included here with a maximum of three iterations per CG step, to find the minimum along the direction of descent.

\section{APPLICATION OF BEM TO ELASTOGRAPHY}

The main subject of investigation was chosen to be a $7 \mathrm{~cm}$ square plate, as a close estimate to the cross sectional size of a human breast. Simulated mechanical parameters, if not subjected to variation, were chosen similar to those of soft tissue with a density of $\rho=1020 \mathrm{~kg} / \mathrm{m}^{3}$, Young's Modulus between $E=20 \mathrm{kPa}$ and $30 \mathrm{kPa}$ and a Poisson's Ratio chosen between $\nu=0.45$ and 0.5 at values approaching incompressibility. 
In all static testing, with or without inclusion, the plate was subjected boundary conditions simulating a tensile test with a longitudinal strain of $10 \%$. In the dynamic case, the plate was subjected to a sinusoidal actuation at the bottom of the plate only, with an amplitude of $1 \mathrm{~mm}$ and a range of frequencies between $30 \mathrm{~Hz}$ and $100 \mathrm{~Hz}$. Figure 1 shows the representative results of a static tensile test with poisson's ratio chosen $\nu=0.5$ for incompressible conditions, as well as the results from harmonic actuation at $50 \mathrm{~Hz}$.

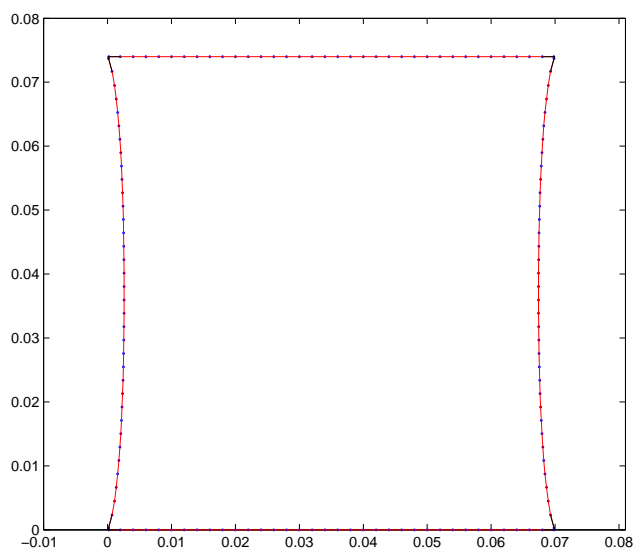

(a)

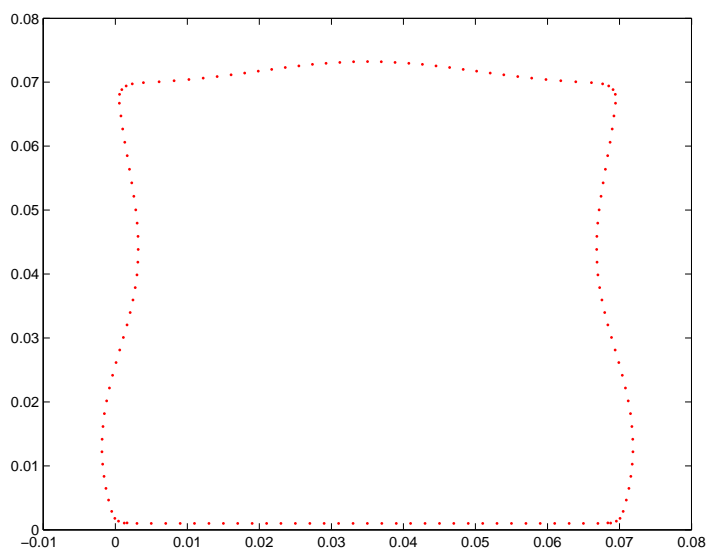

(b)

Figure 1. BEM results for a square plate $(7 \mathrm{~cm} \times 7 \mathrm{~cm}),(a)$ meshed with linear elements and discontinuous elements at the corners under tensile test conditions, and $(b)$ meshed with constant elements and rounded corners, harmonically actuated at $50 \mathrm{~Hz}$ at the bottom of the plate.

Results on simulated inverse problems are presented in the following subsections.

\subsection{Single Homogeneous Region}

The first inverse Problem was carried out for the reconstruction of Poisson's Ratio of a single homogeneous region, as also shown in Figure 1(a), which was simulated at $\nu=0.3$. The start value in the CG iterations was set to $\nu_{0}=0.5$. The inverse solver converged after 200 iterations to a value of $\nu_{\text {conv }}=0.303$ with only $1 \%$ relative error to the original simulated parameter. The convergence plot is shown in figure $2(a)$.

The recovery of elasticity was simulated using a square plate under harmonic actuation, as shown also in Figure $1(b)$ with Poisson's Ration set to $\nu=0.45$ and $E=20 k P a$. An estimate for the start value for E was chosen at $E_{0}=30 \mathrm{kPa}$. Figure $2(\mathrm{~b})$ shows that only six iterations were required to converge to the correct value of $E$ (at an error of $0.08 \%$ ).

Further investigations (comp. Table 3) have generally shown that the convergence behavior of the reconstruction process for Poisson's Ratio is more sensitive to a good estimate of the start value than the reconstruction for elastic modulus.

\subsection{Regions with a stiff Inclusions}

Inclusion problems were simulated here for static actuation. For a clearer description in the following section, the outer region will be referred to as region I and the inclusion as region II, regional parameters will be indicated by subscripts. The typical effect of a large inclusion ten times stiffer than the surrounding material and located in a square plate is shown in Figure 3.

Multi-region based simulations contain the option for the reconstruction of the parameter of elastic contrast $\alpha$ between the stiffness of two regions. Setting $\alpha=E_{I I} / E_{I}$ and setting either one of the parameters to a known value, most logically $E_{I}$ being approximately the stiffness value of healthy tissue, the reconstruction of the contrast parameter $\alpha$ is possible. 


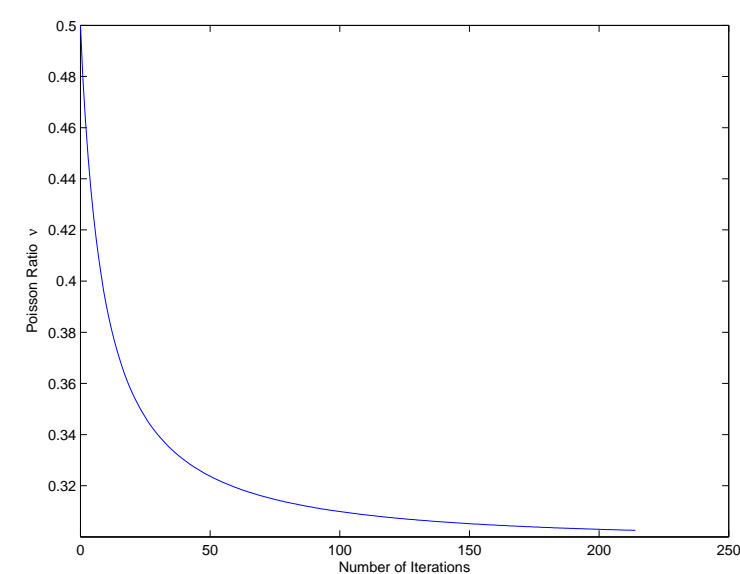

(a)

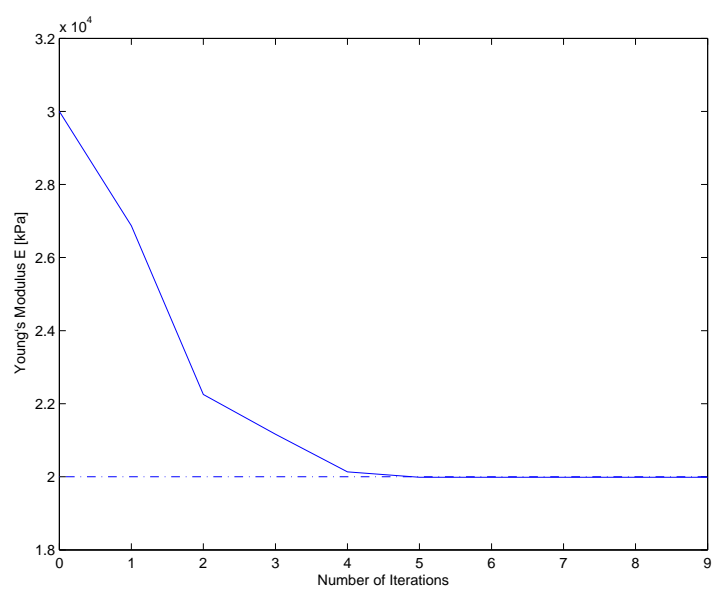

(b)

Figure 2. (a) Convergence of Poisson's Ratio $\nu$ for a square plate under tensile testing, and (b) Convergence of Young's modulus E from a harmonically actuated plate.

Measured data was again obtained by forward simulations with the stiffness of region II chosen ten times stiffer than that one of region I (where $E_{I}=20 \mathrm{kPa}$ ), while Poisson's ratio was set to $\nu_{I}=\nu_{I I}=0.5$. A range of inverse problems has been run reconstructing $\alpha$ with the start value $\alpha_{0}=0.5$, while the size of the inclusion varied from $10 \mathrm{~mm}$ down to $1 \mathrm{~mm}$ in diameter. In all cases, the reconstruction converged within less than eight iterations to the correct value of $\alpha=10$. The convergence behavior was observed to be better in the case of small inclusions. Figure 4 shows the convergence behavior of the reconstruction of $\alpha$ for a small and a large inclusion. For more details, please refer to Appendix B. Simulating the same problem with a change of the inclusion's position within the plate did not lead to significantly different results in convergence.

The simulation of the reversed problem, an inclusion of the same stiffness of region I, therefore a healthy condition, simulated with a start value of $\alpha_{0}=10$ converged in every case to the correct contrast ratio of $\alpha=1$,

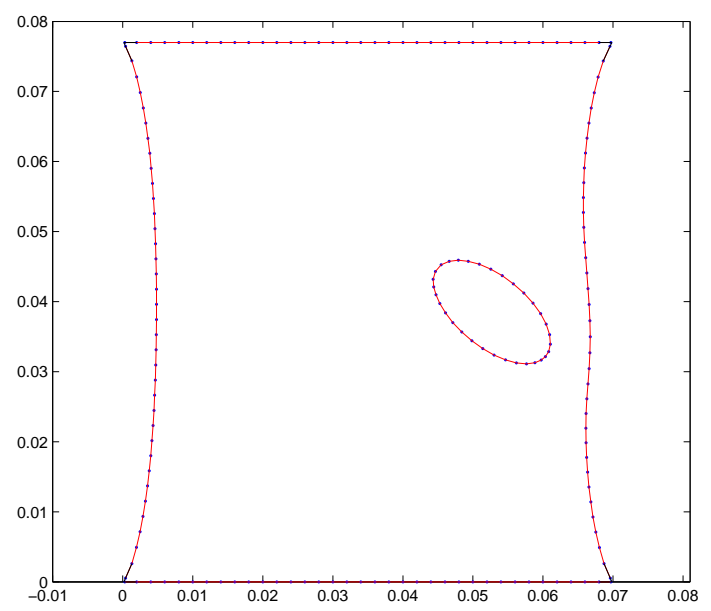

Figure 3. A typical result of a simulation of a square plate with an inclusion under tensile test conditions 


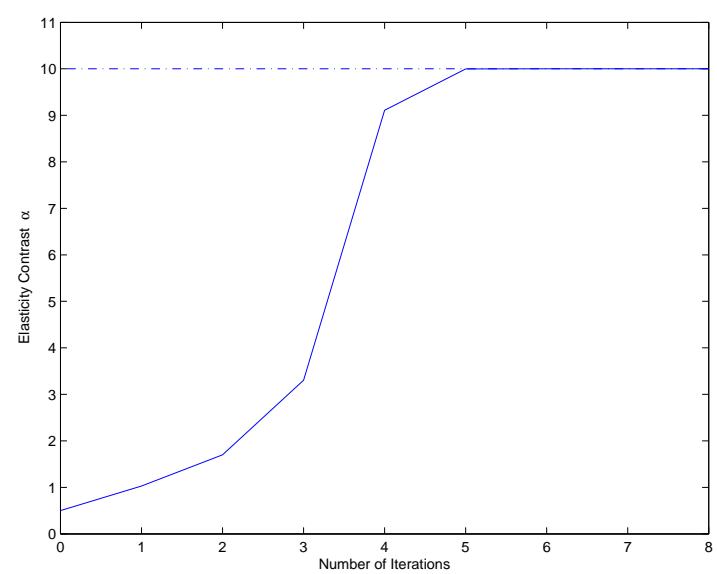

(a)

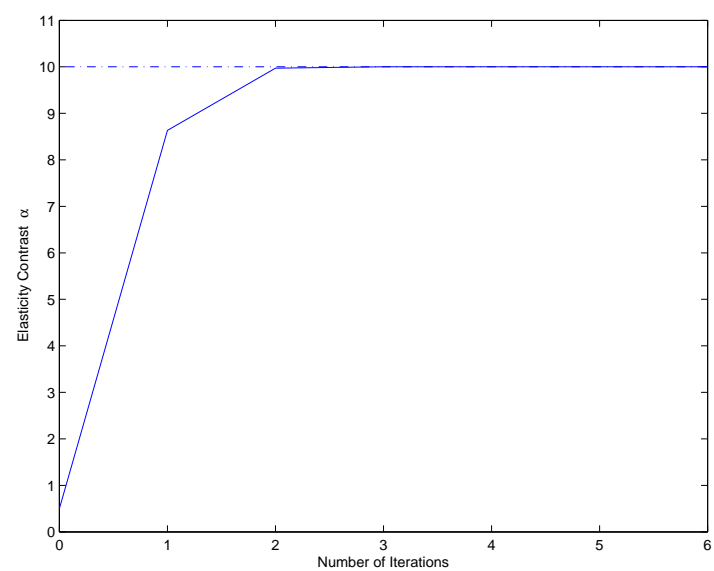

(b)

Figure 4. Convergence of Elasticity Contrast $\alpha$ for a square plate under tensile testing conditions with an inclusion of (a) $10 \mathrm{~mm}$ and (b) $1 \mathrm{~mm}$ in diameter. The correct contrast value is indicated by the dash-dotted line.

as shown in Figure 5 .

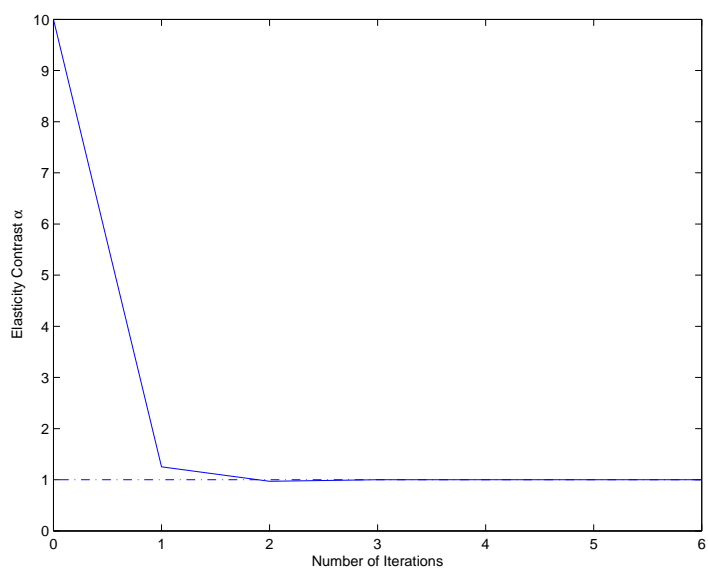

(a)

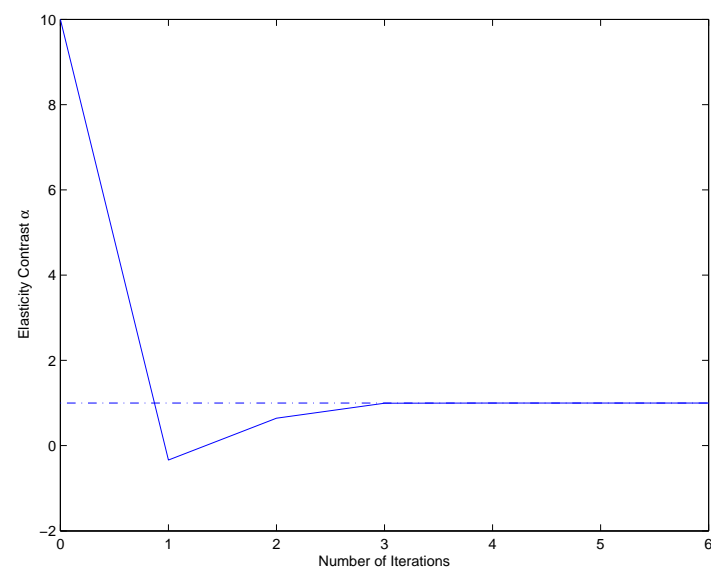

(b)

Figure 5. Convergence of Elasticity Contrast $\alpha$ for a square plate under tensile testing conditions with an inclusion of (a) $10 \mathrm{~mm}$ and $(b) 1 \mathrm{~mm}$ in diameter, but the same elastic Modulus (healthy). The correct contrast value is indicated by the dash-dotted line.

To extend the nature of the problem described above towards more realistic conditions, further simulations were done with uniformly distributed noise added to the measurements of displacements in amounts of up to $10 \%$ of the maximum absolute value of all nodal displacements measured. These simulations still have a good converge behavior, but show a rapid decrease with respect to the quality of the result when increasing the amount of noise.

Most simulations at $5 \%$ added noise converged to contrast values with not more than $30 \%$ relative error, and with no significant difference in the convergence behavior between small or large inclusions. However, simulations carried out at $10 \%$ added noise resulted in much more sensitivity to start values. Although the convergence behavior did not change significantly, convergence occurred with respect to local minima that appeared at 
contrast values with up to $150 \%$ relative error. Also an increase in number of required iterations was generally observed. Figure 6 representatively shows the convergence plots of an inverse problem simulated for an inclusion of $3 \mathrm{~mm}$ in diameter at $5 \%$ and at $10 \%$ added noise. More detailed results on noisy inverse problems carried out are given in Appendix B in tabular form.

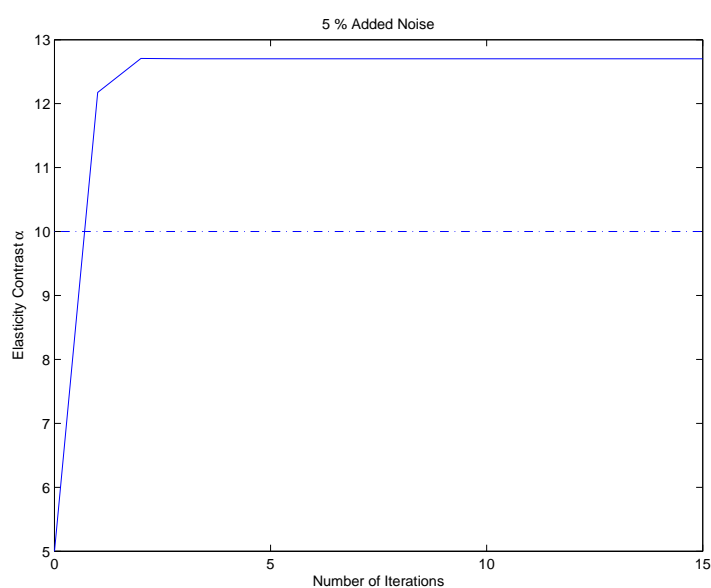

(a)

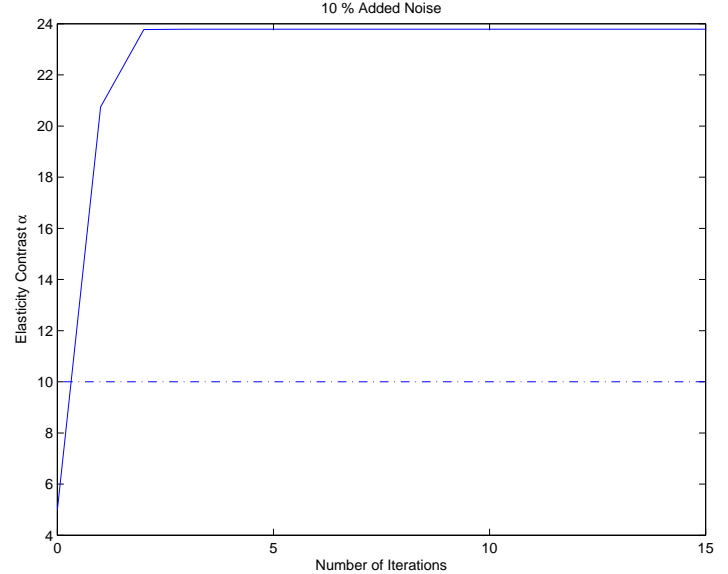

(b)

Figure 6. Convergence of Elasticity Contrast $\alpha$ for a square plate under tensile testing conditions with an inclusion of $3 \mathrm{~mm}$ in diameter at (a) $5 \%$ and (b) $10 \%$ added noise. The correct stiffness contrast value is indicated by the dash-dotted line.

For the sake of completeness, reconstructions for Poisson's Ratio of both regions was performed for a square plate under the same conditions as described above, and with Poisson Ratio's of $\nu_{0_{I}}=0.3$ and $\nu_{0_{I I}}=0.5$. The start values were chosen $\nu_{I}=\nu_{I I}=0.4$ and resulted in a conversion to the correct values of Poisson Ratios with under 20 iterations. The convergence behavior with respect to Poisson's Ratio was overall insignificantly different to that of contrast ratio (comp. Fig. 4), however, more iterations were required in general, especially when the inclusion size was decreased. Some results of these reconstruction problems are presented in Appendix B.

\section{CONCLUSIONS AND FUTURE WORK}

This paper has presented a range of inverse problems that reconstruct the mechanical parameters Poisson's Ratio and Elastic Modulus from measured displacements by the use of either the Boundary Element Method for 2D static or harmonic Elasticity as forward solvers. It was seen that the reconstruction process with a nonlinear CG inverse solver achieved a comparatively fast convergence in all cases, including those with added noise.

It appears that the use of the Boundary Element Method in Elastography and in particular the DIET system has potential to be used as a fast, accurate simulation method.

Future work includes the extension of the method to three-dimensional applications and experimental testing on gelatine phantoms.

\section{APPENDIX A. FUNDAMENTAL SOLUTIONS}

The fundamental solutions to the Elastostatic and Elastodynamic boundary element method, as required in Section 2.1 are given here as displacement and traction solution in tensor notation and polar coordinates for completeness.

In these equations, $r=\sqrt{\left(x_{i}-y_{i}\right)\left(x_{i}-y_{i}\right)}$ is the radius from the collocation point $\mathbf{y}$ to the field point $\mathbf{x}$ with the outward pointing normal vector $n$. The radial derivative with respect to the outward normal can be 
determined using the summation rule as

$$
\frac{\partial r}{\partial n}=\frac{\partial r}{\partial x_{i}} n_{i}, \quad \text { where } \quad \frac{\partial r}{\partial x_{i}}=r_{, i}=\frac{1}{r}\left(x_{i}-y_{i}\right) .
$$

\section{A.1. KELVIN Solution for 2D Elastostatics}

The fundamental solution for 2D Elastostatics is the well known KELVIN-Solution and given as

$$
\begin{gathered}
U_{i j}=\frac{1}{8 \pi \mu(1-\nu)}\left((3-4 \nu) \ln \left(\frac{1}{r}\right) \delta_{i j}+r_{, i} r_{, j}\right) \\
T_{i j}=-\frac{1}{4 \pi(1-\nu) r}\left(\frac{\partial r}{\partial n}\left((1-2 \nu) \delta_{i j}+2 r_{, i} r_{, j}\right)+(1-2 \nu)\left(n_{i} r_{, j}-n_{j} r_{, i}\right)\right) .
\end{gathered}
$$

\section{A.2. Fundamental solution for 2D Elastodynamics}

The fundamental solution for 2D steady state Elastodynamics has got the additional parameters actuation frequency $\Omega$, the shear wave and longitudinal wave speeds

$$
C_{s}=\sqrt{\frac{\mu}{\rho}} \quad \text { and } \quad C_{p}=\sqrt{\frac{\lambda+2 \mu}{\rho}},
$$

and is given as

$$
\begin{gathered}
U_{i j}=\frac{1}{2 \pi \mu}\left(\psi \delta_{i j}-\chi r_{, i} r_{, j}\right) \\
T_{i j}=\frac{1}{2 \pi}\left\{\left(\frac{d \psi}{d r}-\frac{1}{r} \chi\right)\left(\delta_{i j} \frac{\partial r}{\partial n}+r_{, i} n_{j}\right)-\frac{2}{r} \chi\left(n_{i} r_{, j}-2 r_{, i} r_{, j} \frac{\partial r}{\partial n}\right)\right. \\
\left.-2 \frac{d \chi}{d r} r_{, i} r_{, j} \frac{\partial r}{\partial n}+\left(\frac{C_{p}^{2}}{C_{s}^{2}}-2\right)\left(\frac{d \psi}{d r}-\frac{d \chi}{d r}-\frac{1}{r} \chi\right) r_{, j} n_{i}\right\} .
\end{gathered}
$$

The potential functions $\psi$ and $\chi$ given as

$$
\begin{gathered}
\psi=K_{0}\left(\frac{i \Omega r}{C_{s}}\right)+\frac{C_{s}}{i \Omega r}\left\{K_{1}\left(\frac{i \Omega r}{C_{s}}\right)-\frac{C_{s}}{C_{p}} K_{1}\left(\frac{i \Omega r}{C_{p}}\right)\right\} \\
\chi=K_{2}\left(\frac{i \Omega r}{C_{s}}\right)-\frac{C_{s}^{2}}{C_{p}^{2}} K_{2}\left(\frac{i \Omega r}{C_{p}}\right)
\end{gathered}
$$

in terms of $K_{0}, K_{1}$ and $K_{2}$, the modified bessel functions of second kind and order 0,1 and 2, where $i$ is the imaginary unit.

\section{APPENDIX B. TABULATED RECONSTRUCTION RESULTS}

This sections present some detailed samples of reconstruction results in tabular form, as refered to in the text, but however is not understood statistically representative. 
Table 1. Reconstruction of Elasticity Contrast $\alpha$ under $0 \%$ added Noise

\begin{tabular}{|c|c|c|c|c|}
\hline Inclusion $\varnothing[\mathrm{mm}]$ & $\alpha_{0}$ & $\#$ of Iterations & converged to $\alpha=$ & rel. Error [\%] \\
\hline 1 & 0.5 & 4 & 10 & 0 \\
\hline 2 & 0.5 & 4 & 10 & 0 \\
\hline 3 & 0.5 & 4 & 10 & 0 \\
\hline 5 & 0.5 & 5 & 10 & 0 \\
\hline 10 & 0.5 & 7 & 10 & 0 \\
\hline
\end{tabular}

Table 2. Reconstruction of Elasticity Contrast $\alpha$ under $5 \%$ added Noise

\begin{tabular}{|c|c|c|c|c|}
\hline Inclusion $\varnothing[\mathrm{mm}]$ & $\alpha_{0}$ & \# of Iterations & converged to $\alpha=$ & rel. Error [\%] \\
\hline 1 & 0.5 & 4 & 9.51 & 5.1 \\
\hline 2 & 0.5 & 4 & 12.71 & 27.1 \\
\hline 3 & 0.5 & 4 & 12.78 & 27.8 \\
\hline 5 & 0.5 & 4 & 11.83 & 18.3 \\
\hline 10 & 0.5 & 7 & 12.91 & 29.1 \\
\hline
\end{tabular}

Table 3. Reconstruction of Elasticity Contrast $\alpha$ under $10 \%$ added Noise

\begin{tabular}{|c|c|c|c|c|}
\hline Inclusion $\varnothing[\mathrm{mm}]$ & $\alpha_{0}$ & \# of Iterations & converged to $\alpha=$ & rel. Error [\%] \\
\hline 1 & 5 & 7 & 20.5 & 105 \\
\hline 2 & 5 & 5 & 22.6 & 126 \\
\hline 3 & 5 & 6 & 23.8 & 138 \\
\hline 5 & 5 & 6 & 12.6 & 26 \\
\hline 10 & 5 & 9 & 7.4 & 26 \\
\hline
\end{tabular}

Table 4. Reconstruction of Poisson's Ratio $\nu_{I}$ and $\nu_{I I}$ of an inclusion problem under $0 \%$ added Noise

\begin{tabular}{|c|c|c|c|c|c|c|}
\hline Inclusion $\varnothing[\mathrm{mm}]$ & $\nu_{0_{I}}$ & $\nu_{0_{I I}}$ & \# of Iterations & conv. to $\nu_{I}=$ & conv. to $\nu_{I I}=$ & rel. Error [\%] \\
\hline 1 & 0.4 & 0.4 & 23 & 0.3 & 0.5 & 0 \\
\hline 2 & 0.4 & 0.4 & 23 & 0.3 & 0.5 & 0 \\
\hline 3 & 0.4 & 0.4 & 19 & 0.3 & 0.5 & 0 \\
\hline 5 & 0.4 & 0.4 & 17 & 0.3 & 0.5 & 0 \\
\hline 10 & 0.4 & 0.4 & 17 & 0.3 & 0.5 & 0 \\
\hline
\end{tabular}

\section{REFERENCES}

1. T. Suzuki, M. Toi, S. Saji, K. Horiguchi, T. Aruga, E. Suzuki, S. Horiguchi, N. Funata, K. Karasawa, and N. Kamata, "Early breast cancer," Int. J. Clin. Oncol 11, pp. 108-119, 2006.

2. N. Z. H. I. Service, "Cancer: New registrations and deaths 1999," New Zealand Ministry of Health, 2002. 
3. V. C. Cokkinides, A. Samuels, E. M. Ward, and M. J. Thun, Cancer Prevention EG Early Detection Facts Es Figures, American Cancer Society, 2004.

4. L. Tabar, M. F. Yen, V. B., H. Chen, R. Smith, and S. Duffy, "Mammography service screening and mortality in breast cancer patients: 20-year follow-up before and after introduction of screening," Lancet 361(9367), pp. 1405-1410, 2003.

5. D. B. Kopans, Breast Imaging, Lippincott-Ravens, Philadelphia, PA, USA, second ed., 1998.

6. S. Gupta, "A new breast cancer test." http://www.time.com/time/magazine/article/0,9171,1565516,00.html.

7. T. A. Krouskop, T. M. Wheeler, F. Kallel, B. S. Garra, and T. Hall, "Elastic moduli of breast and prostate tissues under compression," Ultrasonic Imaging 20(4), pp. 260-274, 1998.

8. A. Samani, J. Bishop, C. Luginbuhl, and D. B. Plewes, "Measuring the elastic modulus of ex vivo small tissue samples," Physics in Medicine and Biology 48, pp. 2183-2198, 2003.

9. D. B. Plewes, J. Bishop, A. Samani, and J. Sciarretta, "Visualization and quantification of breast cancer biomechanical properties with magnetic resonance elastography," Phys Med Biol 45(6), pp. 1591-610, 2000.

10. M. M. Doyley, J. B. Weaver, E. E. W. Van Houten, F. E. Kennedy, and K. D. Paulsen, "Thresholds for detecting and characterizing focal lesions, using steady-state mr elastography," Medical Physics 30, pp. 495-504, 2003.

11. J. Bercoff, S. Chaffai, M. Tanter, L. Sandrin, S. Catheline, M. Fink, J. L. Gennisson, and M. Meunier, "In vivo breast tumor detection using transient elastography," Ultrasound in Medicine and Biology 29(10), pp. $1387-1396,2003$.

12. E. E. W. Van Houten, M. I. Miga, J. B. Weaver, F. E. Kennedy, and K. D. Paulsen, "Three-dimensional subzone-based reconstruction algorithm for $\mathrm{mr}$ elastography," Magnetic Resonance in Medicine 45(5), pp. 827-837, 2001.

13. E. E. W. Van Houten, M. M. Doyley, F. E. Kennedy, J. B. Weaver, and K. D. Paulsen, "Initial in vivo experience with steady-state subzone-based MR elastography of the human breast," Journal of Magnetic Resonance Imaging 17(1), pp. 72-85, 2003.

14. J. G. Chase, E. E. W. Van Houten, L. Ray, D. Bates, J. P. Henderson, C. Ewing, and C. Berg, "Digital image-based elasto-tomography for soft tissue imaging," in Proceedings of the 1st Asian Pacific Conference on Biomechanics, 2004.

15. A. Peters, A. Milsant, J. Rouze, L. Ray, J. G. Chase, and E. E. W. Van Houten, "Digital-image based elasto-tomography: Proof of concept studies for surface based mechanical property reconstruction," JSME International Journal 47(4), pp. 1117-1123, 2004.

16. A. Peters, S. Wortmann, R. Elliott, M. Staiger, J. G. Chase, and E. E. W. Van Houten, "Digital imagebased elasto-tomography: First experiments in surface based mechanical property estimation of gelatine phantoms," JSME International Journal 48(4), 2005.

17. A. Peters, H.-U. Berger, J. G. Chase, and E. E. W. Van Houten, "Digital image-based elasto-tomography: Nonlinear mechanical property reconstruction of homogeneous gelatine phantoms," International Journal of Information and System Sciences 2(4), pp. 512-521, 2006.

18. P. K. Banerjee, The Boundary Element Methods in Engineering, McGraw-Hill, second ed., 1981.

19. C. A. Brebbia and J. Dominguez, Boundary Elements - An introductory Course, McGraw-Hill, 1992.

20. G. Beer, Programming the Boundary Element Method - An Introduction for Engineers, Wiley, 2001.

21. G. D. Manolis and D. E. Beskos, Boundary Element methods in Elastodynamics, Allen and Unwyn, 1988.

22. J. Dominguez and E. Alarcon, Elastodynamics, vol. 1, ch. 7, pp. 213-257. Pentech Press, London, 1981.

23. S. Ahmad and P. Banerjee, "Multi-domain bem for two-dimensional problems in elastodynamics," International Journal for Numerical Methods in Engineering 26, pp. 891-911, 1988. 